\title{
Prognosis of rectal neuroendocrine tumors after endoscopic resection: a single-center retrospective study
}

\author{
Yue Zheng ${ }^{1 \#}$, Kehang Guo ${ }^{1,2 \#}$, Ruijie Zeng ${ }^{1,3 \#}$, Zhendao Chen $^{4 \#}$, Wanwei Liu ${ }^{1}$, Xiaoguang Zhang ${ }^{1}$, \\ Weimin Liang ${ }^{1}$, Jianhua Liu ${ }^{5}$, Hao Chen ${ }^{1}$, Weihong Sha ${ }^{1}$
}

${ }^{1}$ Department of Gastroenterology, Guangdong Provincial People’s Hospital, Guangdong Academy of Medical Sciences, Guangzhou, China; ${ }^{2}$ School of Medicine, South China University of Technology, Guangzhou, China; ${ }^{3}$ Shantou University Medical College, Shantou, China; ${ }^{4}$ Department of Gastroenterology, Huazhou People's Hospital, Maoming, China; 'Department of Oncology, Cancer Center, Guangdong Provincial People's Hospital, Guangdong Academy of Medical Sciences, Guangzhou, China

Contributions: (I) Conception and design: Y Zheng, J Liu, H Chen, W Sha; (II) Administrative support: W Liu, X Zhang, W Liang; (III) Provision of study materials or patients: Y Zheng, X Zhang; (IV) Collection and assembly of data: Y Zheng, K Guo, R Zeng, Z Chen; (V) Data analysis and interpretation: Y Zheng, K Guo, R Zeng; (VI) Manuscript writing: All authors; (VII) Final approval of manuscript: All authors.

\#These authors contributed equally to this work.

Correspondence to: Prof. Weihong Sha. Department of Gastroenterology, Guangdong Provincial People's Hospital, Guangdong Academy of Medical Sciences, Guangzhou 510080, China. Email: shaweihong@gdph.org.cn; Prof. Hao Chen. Department of Gastroenterology, Guangdong Provincial People’s Hospital, Guangdong Academy of Medical Sciences, Guangzhou 510080, China. Email: chenhao@gdph.org.cn; Dr. Jianhua Liu. Department of Oncology, Cancer Center, Guangdong Provincial People's Hospital, Guangdong Academy of Medical Sciences, Guangzhou 510080, China. Email: liujianh80@126.com.

Background: The efficacy of endoscopic resection in patients with rectal neuroendocrine tumors (NETs) which are less than $20 \mathrm{~mm}$ in diameter remains unclear. This study aimed to investigate the efficacy and outcomes of different types of endoscopic resection in patients with NETs.

Methods: We performed a retrospective analysis and follow-up on 98 patients who underwent endoscopic resection for rectal NETs between August 2010 and October 2019 at Guangdong Provincial People's Hospital, China. The lesions were preoperatively classified according to their endoscopic morphology and measured by endoscopic ultrasound. Patients were divided into modified endoscopic mucosal resection (EMR) and endoscopic submucosal dissection (ESD) groups depending on the endoscopic treatment they received. The en bloc resection rate, histopathological complete resection rate, and the complication rate of the 2 groups were evaluated after the operation. The risk factors for incomplete resection were also analyzed.

Results: The average diameter of the 98 NETs was $6.29 \pm 2.90 \mathrm{~mm}$ (range, $2-15 \mathrm{~mm}$ ). The en bloc resection rate of the modified EMR and ESD treatment groups was $97.2 \%(35 / 36)$ and $100 \%(62 / 62)$, respectively. The histopathological complete resection rate was $86.1 \%$ (31/36) and 87.1\% (54/62), respectively. No tumor recurrence or tumor-related death occurred. There were no statistically significant differences in the rate of histopathological complete resection, perforation, or delayed hemorrhage between the 2 groups $(\mathrm{P}>0.05)$. Multivariate analysis demonstrated that the depth of tumor invasion $(\mathrm{P}=0.007)$ and tumor diameter $(\mathrm{P}<0.001)$ were independent risk factors for histopathological complete resection.

Conclusions: Modified EMR and ESD are safe and effective endoscopic approaches for the resection of rectal NETs $\leq 15 \mathrm{~mm}$ in diameter. Endoscopic resection requires a comprehensive preoperative evaluation of risk factors including the depth of tumor invasion and tumor diameter.

Keywords: Rectal neuroendocrine tumor; endoscopic resection; efficacy; safety

Submitted Jul 08, 2021. Accepted for publication Nov 24, 2021.

doi: 10.21037/jgo-21-391

View this article at: https://dx.doi.org/10.21037/jgo-21-391 


\section{Introduction}

Rectal neuroendocrine tumors (NETs) are small but potentially malignant tumors, which were once considered rare. However, due to the rapid prevalence of colonoscopy in the past few decades, the global incidence of rectal NETs has increased by several to a dozen times, while the detection rate via colonoscopy is about $0.05-0.07 \%$ (1-6). In Asia, the rectum is the most common primary site for gastroenteropancreatic NETs, of which rectal NETs account for $60-89 \%$. The prognosis of rectal NETs can vary greatly depending on tumor grade, size, depth of invasion, and lymphatic vascular invasion (7-10), which has raised increasing concern among clinicians. It has been noted that $66-80 \%$ of rectal NETs are small in size and found incidentally under colonoscopy (2). Currently, in situ tumors $\leq 10 \mathrm{~mm}$ in diameter and confined to the mucosa and submucosa are acknowledged to have a low risk of lymph node metastases and can be treated locally with endoscopic resection (11). Endoscopic treatment approaches include conventional endoscopic mucosal resection (EMR), modified EMRs such as cap-assisted EMR (EMR-C) and ligation-assisted endoscopic mucosal resection (EMR-L), and endoscopic submucosal dissection (ESD) $(12,13)$. Currently, most guidelines recommended modified EMR or ESD for the endoscopic treatment of rectal NETs to improve histologically complete resection of the lesion and to reduce the rate of residual tissue $(14,15)$. However, recent studies have reported a $10-15 \%$ risk of metastases for tumors $11-20 \mathrm{~mm}$ in diameter, and the efficacy of endoscopic resection remains unclear $(5,16)$. In addition, the therapeutic efficacy of different endoscopic resection approaches may differ. To this end, this study retrospectively analyzed 98 patients who underwent endoscopic resection for rectal NETs in our hospital from August 2010 to October 2019. The aim was to investigate the efficacy of different types of endoscopic resection by analyzing the tumor morphology along with the complete resection rate, complication rate, and outcomes. We present the following article in accordance with the Strengthening the Reporting of Observational Studies in Epidemiology (STROBE) reporting checklist (available at https://dx.doi. org/10.21037/jgo-21-391).

\section{Methods}

\section{Study participants}

From August 2010 to October 2019, a total of 159 patients with rectal NETs underwent endoscopic resection in Guangdong Provincial People's Hospital. Among them, 98 were included in this study, while the rest were excluded for various reasons (38 underwent conventional EMR and 11 underwent preoperative biopsy, while in 12 cases, the lesion margins were not successfully assessed). The inclusion criteria were as follows: (I) patients were clinically diagnosed with rectal NETs by ultrasound colonoscopy, and the lesions were confined to the mucosal or submucosal layer with a maximum diameter of $20 \mathrm{~mm}$; (II) patients underwent an enhanced computed tomography (CT) scan of the chest and the whole abdomen before surgery to exclude lymph node and distant metastases; and (III) the diagnosis of the NET was confirmed by hematoxylin and eosin (HE) staining and immunohistochemical (IHC) staining. The exclusion criteria were as follows: (I) the tumor was larger than $20 \mathrm{~mm}$ in diameter, or with ulceration or bleeding on the surface; (II) patients had distant metastases; (III) patients had a rectal NET but underwent endoscopy or biopsy in another hospital; (IV) the lesion demonstrated a nonlifting sign after submucosal injection before surgery; (V) patients' NETs were connected to another site in the digestive tract; and (VI) the follow-up time was less than 6 months (Figure 1).

\section{Endoscopic classification of tumors}

The endoscopic classification of tumors consisted of 3 types: type I, flat lesions less than $2.5 \mathrm{~mm}$ in height (smaller than the diameter of the closed biopsy forceps); type II, dome-shaped or hemispherical lesions larger than or equal to $2.5 \mathrm{~mm}$ (the diameter of the closed biopsy forceps); and type III, lesions with visible depression. The morphological classification of all cases was independently assessed by 2 experienced endoscopists and finally determined by consensus.

\section{Surgical procedures}

For preoperative preparation: UM2000 7.5/12 MHz ultrasound probes (Olympus Corporation, Tokyo, Japan) were used to assess tumor size and the depth of invasion before surgery. All operations were performed independently by highly experienced endoscopists in our hospital. Both EMR-C and ESD (GIF-Q260J and GIF-Q260, respectively; Olympus Corporation) were performed using single-channel endoscopes (each of which had a transparent cap fitted on the top) and VIO200D high-frequency electrosurgical devices (ERBE, Tübingen, 
A total of 159 patients with rectal NETs underwent endoscopic resection in Guangdong Provincial People's Hospital from August 2010 to October 2019

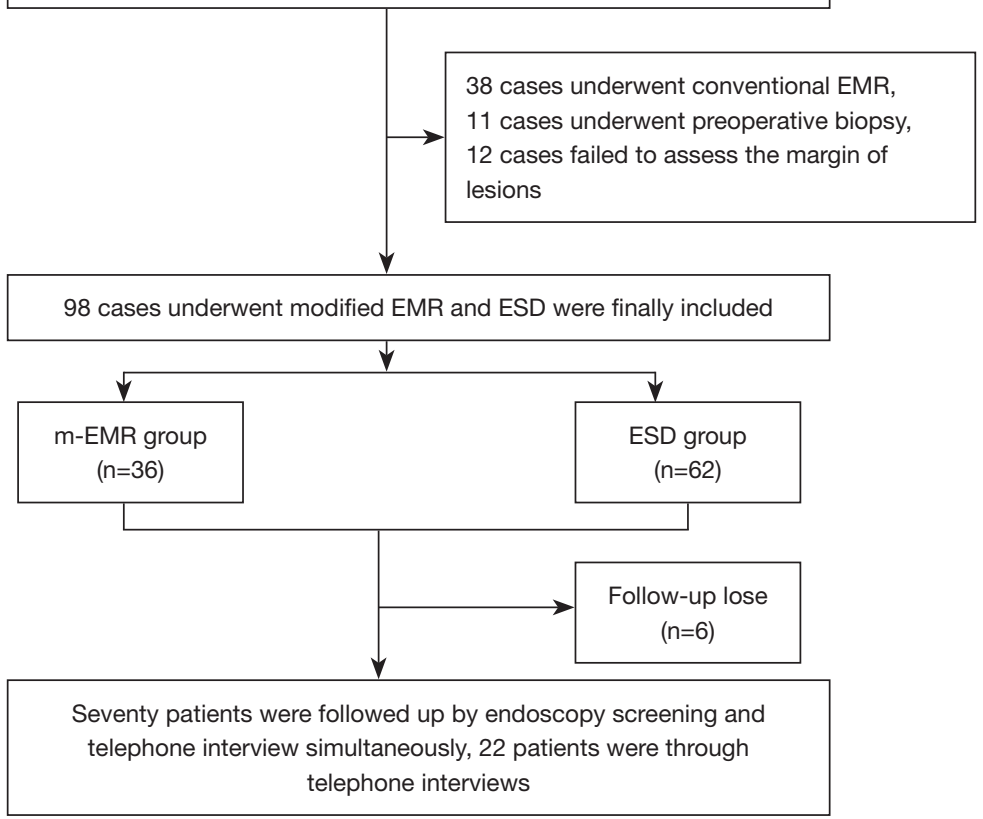

Flow chart showing the inclusion of study patients

Figure 1 Flowchart of the inclusion of patients in this study.
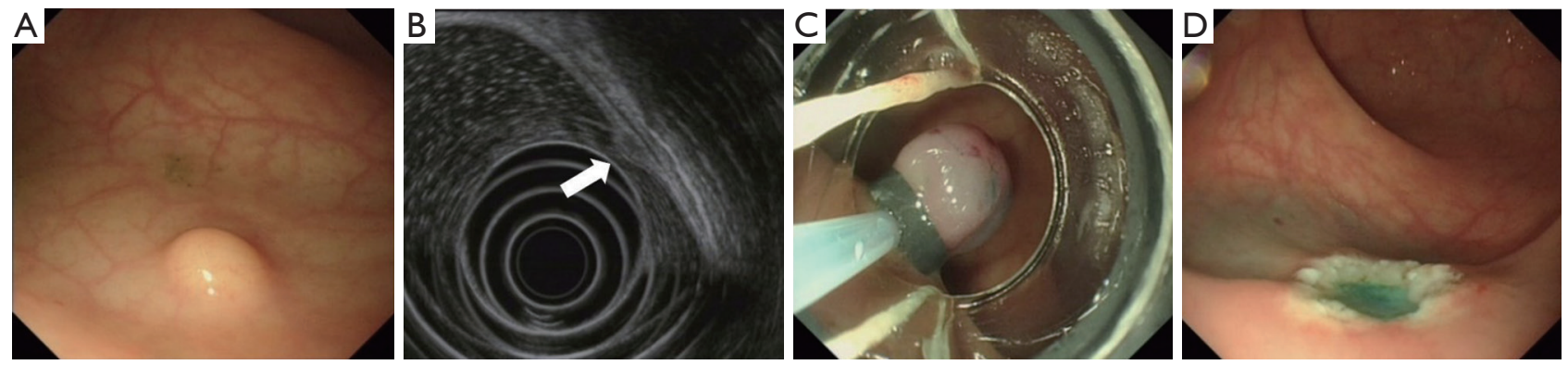

Figure 2 EMR-L of rectal neuroendocrine tumor. (A) Rectal tumor at the initial colonoscopy. (B) Ultrasound endoscopy showing tumor confined to the submucosa. (C) Aspiration of the lesion into the transparent cap and release of the ligature ring. (D) Complete resection of lesion. White arrow: tumor. EMR-L, ligation-assisted endoscopic mucosal resection; NET, neuroendocrine tumor.

Germany). EMR-L was performed with ligation devices, and polypectomy snares (Cook Medical LLC, Bloomington, IN, USA) were used to remove the lesions after ligation. Dual knives and/or IT knives (Olympus Corporation, Tokyo, Japan) were used in ESD. Hemostatic forceps were used in the operation to prevent bleeding.

The operating procedures were as follows: (I) for EMR-L, the margin of the lesion was first marked with an argon knife, which was followed by the submucosal injection of normal saline. After fixing a multiple band ligator on the side of the mirror, the endoscopist sucked the lesion into the transparent cap and released the ligator. The lesion was subsequently removed through electrocoagulation (Figure 2). (II) For EMR-C, the margin of the lesion was first marked with an argon knife, which was followed by the submucosal injection of normal saline. After a transparent cap was mounted on the side of the mirror, the lesion was sucked into the transparent cap and 

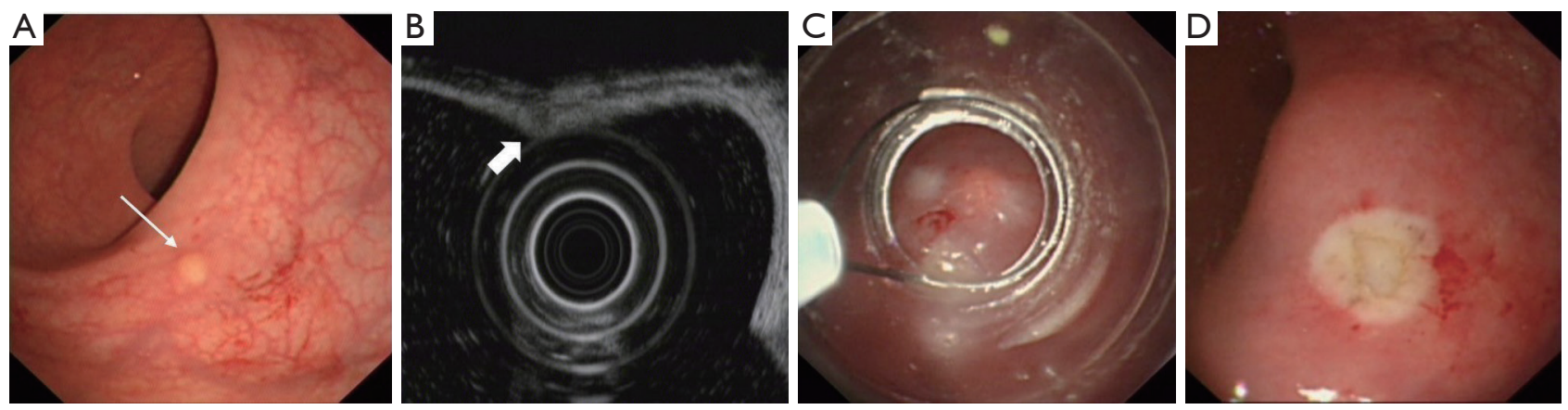

Figure 3 EMR-C of rectal NET. (A) Rectal tumor is seen in the rectum on endoscopy. White arrow: tumor. (B) The submucosal hypoechoic lesion is showed by ultrasound endoscopy. White arrow: tumor. (C) The lesion is pulled into a transparent cap. (D) The lesion of resection is completed. EMR-C, cap-assisted EMR; NET, neuroendocrine tumor.
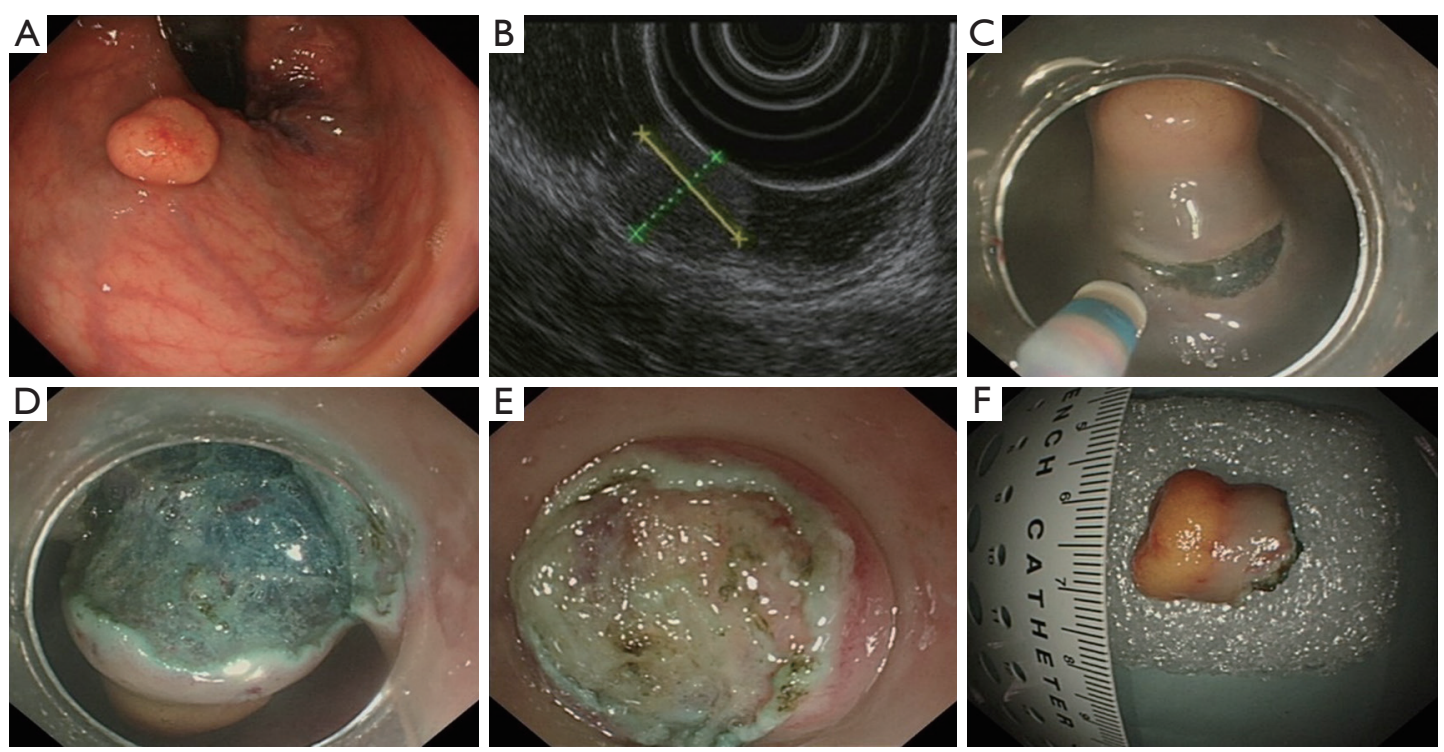

Figure 4 ESD of rectal NET. (A) Rectal tumor. (B) Ultrasound endoscopy showing deep mucosal hypoechoic lesion with posterior echogenic attenuation. (C) Circumferential dissection of the mucosa surrounding the lesion. (D) Partial peeling of the lesion. (E) Complete peeling of the lesion. (F) The resected tumor. ESD, endoscopic submucosal dissection; NET, neuroendocrine tumor.

ligated. Subsequently, the lesion was removed through electrocoagulation (Figure 3). (III) For ESD, submucosal injection of glycerol fructose-methylene blue-adrenaline solution was arranged, and then the mucosa surrounding the lesion was resected circumferentially and peeled off gradually with electrocoagulation until the lesion was completely removed. The wound was thereafter treated with an argon knife (Figure 4).

\section{Study outcomes}

Endoscopic resection specimens were collected to evaluate the en bloc resection rate and histopathological complete resection rate (H-CR) of the lesions. En bloc resection refers to the endoscopic excision of the entirety of a lesion to gain a single specimen. H-CR is defined as the absence of residual tumor on the lateral and deep margins 
of the resected specimen at microscopic examination. Histopathological incomplete resection (H-IR) refers to the presence of residual tumor on the lateral and/or deep margins of the resected specimen at microscopic examination. The size, surgical margin, invasion depth, vascular invasion, and histological grade of tumor were assessed based on HE staining, IHC staining, and other methods. According to the WHO Classification of Digestive System Tumors (2019) (17), we assessed the histological grade (G1, G2, or G3) based on the mitoses and Ki-67 index of the tumor: G1, mitoses $<2 / 10$ HPF (highpower filed) and Ki-67 index $\leq 3 \%$; G2, mitoses $=2-20 / 10$ $\mathrm{HPF}$ and Ki-67 index 3-20\%; and G3, mitoses $>20 / 10 \mathrm{HPF}$ and Ki-67 index $>20 \%$. Vascular invasion was evaluated using D2-40 and CD34 IHC stains.

Complications related to endoscopic treatment included postoperative perforation and delayed postoperative hemorrhage. Postoperative perforation refers to the perforation that is detected postoperatively through endoscopic examination or imaging modalities. Delayed postoperative hemorrhage refers to a hemorrhage that cannot spontaneously stop within $24 \mathrm{~h}$ after endoscopic resection and requires additional clinical interventions.

\section{Postoperative treatment and follow-up}

For patients who underwent complete tumor resection, we recommended a colonoscopy examination every 6 months in the first year after the operation. If there were no signs of recurrence, an annual colonoscopy examination was then recommended. For patients who underwent complete resection but had a vascular invasion, or for patients who underwent incomplete resection and refused additional surgery, a colonoscopy was recommended 3, 6, and 12 months after the operation, and once a year thereafter if no local recurrence occurred. If any suspected recurrence was detected through a colonoscopy follow-up, a biopsy was recommended. In this study, we aimed to follow-up with all patients by endoscopy examination or telephone interview.

\section{Statistical analyses}

Statistical analyses were performed using SPSS 25.0 software (IBM, Armonk, NY, USA). Numeration data were compared with a $\chi^{2}$ test or Fisher's exact test, and measurement data are presented as mean \pm standard deviation. The univariate analyses were performed using a $t$-test or Mann-Whitney $\mathrm{U}$ test. A binary logistic regression analysis was performed to identify risk factors of $\mathrm{H}-\mathrm{CR}$, and each odds ratio (OR) was calculated with a $95 \%$ confidence interval (CI). A P value $<0.05$ was considered statistically significant.

\section{Ethical statement}

The study was conducted in accordance with the Declaration of Helsinki (as revised in 2013). The study was approved by the ethics board of the Guangdong Provincial People's Hospital (No. KY-Q-2021-184-01). Informed consent was waived because of the retrospective nature of the study, and anonymous clinical data was use.

\section{Results}

\section{Baseline characteristics}

A total of 98 patients with rectal NETs were included in this study, including 66 males $(67.3 \%)$ and 32 females (32.7\%). The average age of patients was $48.29 \pm 12.11$ years. The average tumor diameter was $6.29 \pm 2.90 \mathrm{~mm}$. There were 11 tumors located in the lower rectum (less than $5 \mathrm{~cm}$ from the anal margin), 76 tumors located in the middle rectum (5-9 $\mathrm{cm}$ from the anal margin), and 11 tumors located in the upper rectum (10-15 cm from the anal margin).

\section{Endoscopic tumor morphology}

The lesions were round and flat, dome-shaped, or hemispherical. The surface of the mucosa was smooth and complete. The color of the lesions was yellow, pale yellow, or white. Furthermore, 93 patients had a single rectal lesion, and 5 patients had synchronous or multiple rectal lesions. Endoscopic ultrasound (EUS) examination showed a low-to-medium, even, or uneven echo lesion with clear boundaries in the second and/or third layer. Of these, 46 cases were limited to the first or second layers, while 52 cases were limited to the second or third layers (Table 1).

\section{Endoscopic resection}

All 98 patients underwent endoscopic treatment, with 62 undergoing ESD, 14 undergoing EMR-L, and 22 undergoing EMR-C. One patient experienced a delayed postoperative hemorrhage, which was successfully controlled after endoscopic interventions, and no intraoperative perforation occurred. There were no significant differences 
Table 1 The characteristics of patients with rectal neuroendocrine tumors (NETs)

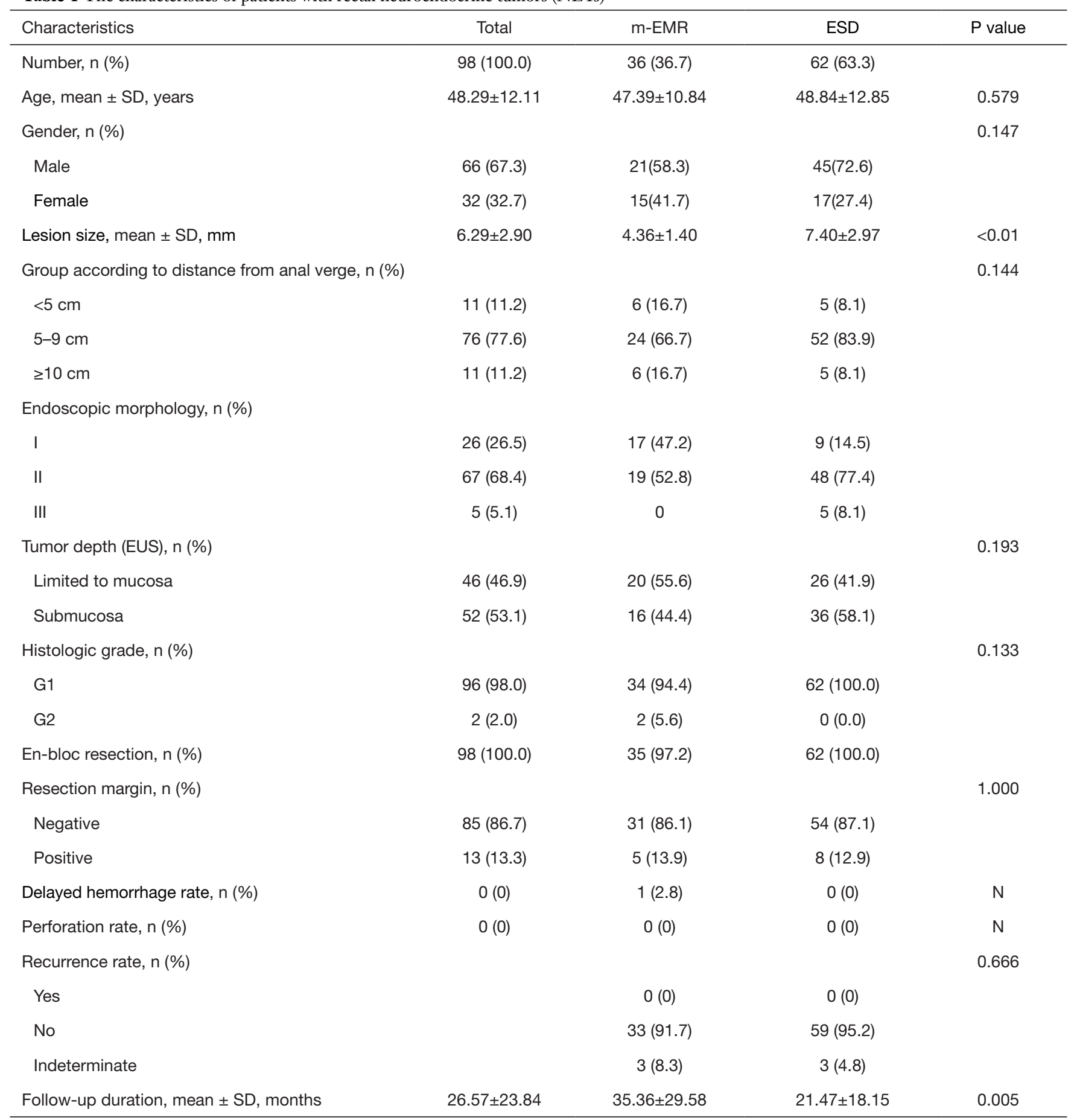


in the incidence of perforation and delayed hemorrhage between the modified EMR and ESD treatment groups ( $\mathrm{P}>0.05$; Table 1).

\section{Histopathological manifestations}

According to the mitoses and $\mathrm{Ki}-67$ proliferation index classification, 96 cases were classified as G1 $(98.0 \%)$ and 2 cases were classified as G2 (2.0\%). The en bloc resection rate was $99.0 \%$ (97/98). There were 85 cases with negative lateral and deep margins and 13 cases with positive surgical margins, resulting in an $\mathrm{H}-\mathrm{CR}$ rate of $86.7 \%$ (85/98). Three patients with positive tumor margins had additional surgery (two with positive vertical margins and 1 with positive lateral and vertical margins), and no residual tumor cells were found at postoperative microscopic examination; 9 of the remaining 10 cases had positive vertical margins, while 1 had a positive lateral and vertical margin. Vascular invasion was found in 3 patients. The lesions were confined to the mucosal layer in 36 cases and had invaded into the mucosal and submucosal layer in 62 cases. There was no statistical difference in the H-CR between the modified EMR and ESD treatment groups $(\mathrm{P}>0.05$; Table 1$)$.

Univariate analyses showed that the H-CR of both ESD and modified EMR was significantly associated with invasion depth, endoscopic classification, and tumor diameter $(\mathrm{P}<0.05)$, while no significant relationship was found between gender, age, distance from the anal margin, surgical procedure, or prognosis (Table 2). In addition, the associations between $\mathrm{H}-\mathrm{CR}$ and invasion depth $(\mathrm{P}=0.007)$ and tumor diameter were highly significant $(\mathrm{P}<0.001$; Table 3$)$.

\section{Follow-up}

We followed up with 70 patients by both endoscopy screening and telephone interview and 22 by telephone interview, but 6 were lost to the follow-up. The follow-up time for the 92 patients ranged from 6 to 98 months, with an average follow-up time of $28.30 \pm 23.58$ months. There was no tumor recurrence or NET-related death.

\section{Discussion}

Gastrointestinal NETs (GI-NETs) are a common type of NET, and the rectum is the most common site of GI-NETs. With the prevalence of colonoscopy, the incidence of rectal NETs has increased rapidly worldwide (18). The differences in tumor grade, size, invasion depth, and lymphatic vascular invasion can directly influence the prognosis of patients and represent a growing challenge among clinicians. According to Ko et al. (19), the risk of developing rectal NETs is 1.5 times more likely to occur in males as opposed to females, and the age of onset ranges from 37 to 57 years old. In the present study, the incidence of rectal NETs was significantly higher in males than in females and highest in middle-aged and elderly patients, which was consistent with Ko et al.'s findings.

According to previous literature, most rectal NETs are located 5-9 $\mathrm{cm}$ away from the anal margin $(20,21)$. In this study, of the 76 cases with tumors located 5-9 $\mathrm{cm}$ from the anal margin, 65 (85.53\%) had tumors less than $10 \mathrm{~mm}$ in diameter. Rectal NETs are small submucosal lesions that can be difficult to detect. For this reason, they can be missed during endoscopic examination if the colonoscopy is withdrawn too quickly. To guarantee the early diagnosis of a tumor and allow more time for treatment, more caution should be taken during rectum colonoscopies, especially for the mid to distal rectum.

Accurate preoperative evaluation of rectal NETs is critical for optimizing treatment decisions and is closely associated with the prognosis of the disease. Guidelines recommend that preoperative EUS examinations be used to assess the tumor size, invasion depth, and lymph node involvement to determine the stage of the disease, followed by the optimal treatment $(22,23)$. It is necessary to perform $\mathrm{CT}$, magnetic resonance imaging (MRI), and other imaging examinations to evaluate local or distant metastases in case of any local lymph node or muscularis propria invasion detected by EUS examination. A previous study showed that the accuracy of EUS in measuring the depth of tumor invasion was $92.5 \%$ (24). The accuracy was $85.7 \%$ (84/98) in this study, which suggests that EUS has a good diagnostic performance in the preoperative evaluation of tumor stage. It is also worth noting that 3 patients with negative surgical margins were found to have vascular invasions in the subsequent pathological evaluation in our study and that the tumor diameter was less than $10 \mathrm{~mm}$. Nagata et al. (25) reported a case of a rectal NET with a diameter of $8 \mathrm{~mm}$ combined with liver metastases. Furthermore, Naunheim et al. (26) found that 13 of 388 patients (3.4\%) with rectal NETs less than $10 \mathrm{~mm}$ in diameter developed metastases, which suggests that patients with tumors smaller than $10 \mathrm{~mm}$ still have a risk of vascular invasion and metastases. In other words, EUS can accurately assess the depth of tumor invasion and significantly improve the accuracy of preoperative local tumor staging. Therefore, despite the 
Table 2 The clinical and clinicopathologic characteristics of the patients with rectal NETs

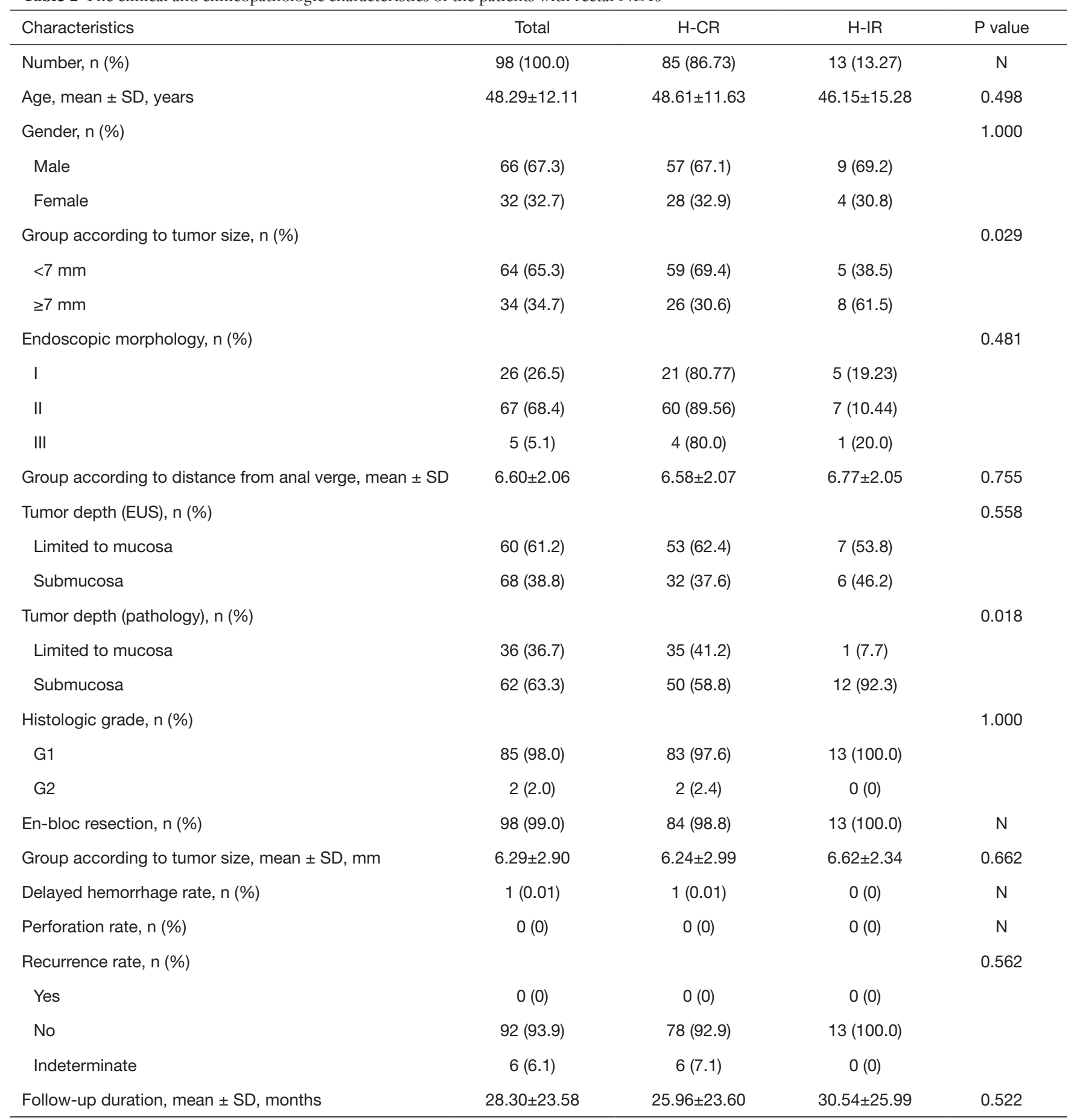

small size of NETs, clinicians should be careful not to overlook the possibility of the tumor developing metastases.

Currently, surgical resection remains the only way to cure rectal NETs. With the rapid development of endoscopic techniques, guidelines are increasingly recommending endoscopic resection be used, as it is simple and convenient while offering reduced trauma and rapid rehabilitation (27-30). According to the international guidelines for 
Table 3 Multivariate analysis to determine factors associated with histologically complete resection

\begin{tabular}{lcc}
\hline Variables & OR $(95 \% \mathrm{Cl})$ & $\mathrm{P}$ value \\
\hline Tumor size & $2.636(1.590-4.369)$ & $<0.001$ \\
Tumor depth (pathology) & 1 (ref) & \\
Limited to mucosa & $4.835(1.534-15.237)$ & 0.007 \\
Submucosa & 1 (ref) & \\
Endoscopic morphology, n (\%) & \\
I & $2.249(0.640-7.898)$ & 0.206 \\
II + III
\end{tabular}

the diagnosis and treatment of gastroenteropancreatic neuroendocrine neoplasms, endoscopic resection is recommended for tumors $<10 \mathrm{~mm}$ in diameter, and endoscopic resection or radical surgery is recommended for tumors of 10-20 mm depending on the invasion depth and lymph node metastases. Endoscopic surgical resection mainly includes conventional EMR, modified EMR, and ESD. Most rectal NETs originate in the deep mucosal and submucosal layers, and have the potential to infiltrate into the submucosa. Conventional EMR techniques can only remove lesions of mucosal origin, for example, colorectal polyps. However, conventional EMR for lesions located in the submucosal layer often results in a high rate of residual lesions and requires further endoscopic or surgical treatment. Therefore, according to the recommendations of most of the current guidelines, a modified EMR or ESD is recommended for rectal NETs to improve the rate of histologically complete resection and to reduce the rate of tissue residue. Due to the potential risk of metastasis of rectal NETs, it is of critical importance to guarantee the $\mathrm{H}-\mathrm{CR}$ of the tumor during endoscopic treatment. Many studies have proven that, for rectal NETs smaller than $10 \mathrm{~mm}$ in diameter, modified EMR affords a higher rate of H-CR than does conventional EMR (13,31). Yang et al. (32) showed that for rectal NETs $6-8 \mathrm{~mm}$ in diameter, there was no significant difference in the H-CR rate between modified EMR and ESD. However, few studies have investigated the efficacy and safety of modified EMR and ESD for treating rectal NETs larger than or equal to $7 \mathrm{~mm}$ in diameter. Our study demonstrated that in the treatment of NETs, there were no significant differences between modified EMR (EMR-C/EMR-L) and ESD in terms of the en bloc resection rate $(97.2 \%$ vs. $86.1 \%), \mathrm{H}-\mathrm{CR}$ rate $(2.8 \%$ vs. $0 \%)$, and complication rate $(87.1 \%$ vs. $0 \%)$, confirming that both treatments displayed good efficacy and safety.

A few previous studies have shown that endoscopic approaches, tumor morphology, size, invasion depth, and pathological grade may be risk factors that affect H-CR. For example, Kim et al. showed that the diameter of rectal NETs is a risk factor affecting the $\mathrm{H}-\mathrm{CR}$ rate regardless of the endoscopic approach (EMR or modified EMR) (16). In addition, Wang et al. (33) determined that endoscopic tumor morphology and pathological tumor grade are risk factors that affect the $\mathrm{H}-\mathrm{CR}$ rate. In our study, multivariate analysis showed that tumor diameter and the depth of invasion were risk factors of the $\mathrm{H}-\mathrm{CR}$ rate (OR $=2.636, \mathrm{P}<0.001 ; \mathrm{OR}=4.835, \mathrm{P}=0.007)$. The risk factors of histological complete endoscopic resection of rectal NETs remain unclear, and more clinical studies are needed to investigate this resection type.

Postoperative follow-up is important in the overall management of rectal NETs and the efficacy assessment of endoscopic treatment. Currently, it is believed that the follow-up strategy after endoscopic resection of rectal NETs should be made upon the recurrence risk, which depends on the tumor size, grade, stage, and other factors. The European Society for Medical Oncology (EMSO) guidelines [2020] do not recommend a follow-up for rectal NETs smaller than $10 \mathrm{~mm}$ in diameter (23). However, Kim et al. (16) reported that among 277 patients undergoing endoscopic resection for rectal NETs, 2 patients had local recurrence and 1 patient died from distant metastasis; all 3 patients had a tumor diameter smaller than $10 \mathrm{~mm}$, and 1 occurred 8 years after surgery. Therefore, the followup strategy for patients with rectal NETs less than $10 \mathrm{~mm}$ in diameter warrants further investigation. For patients with tumors $10-20 \mathrm{~mm}$ in size (G1 or G2), the National Comprehensive Cancer Network (NCCN) and European Neuroendocrine Tumor Society (ENETS) recommend an annual endoscopic follow-up after surgery, and EUS is recommended for further evaluation in case of suspected recurrence. However, several previous studies have demonstrated that patients with rectal NETs smaller than $20 \mathrm{~mm}$ had a good outcome and a low recurrence rate. Sung et al. (34) performed a follow-up of at least 1-year on 157 patients with rectal NETs after endoscopic resection and found that no patients relapsed. Another study showed that no patients developed local tumor recurrence after endoscopic treatment for rectal NETs during a follow-up period of 25.8 to 62.5 months (median: 57.8 months) (35). In this study, there were 13 cases with tumors of $10-20 \mathrm{~mm}$ in diameter (average: $12.3 \pm 2.2 \mathrm{~mm}$ ), and no recurrence 
occurred during the follow-up (31.1 \pm 25.4 months). Singh et al. (36) suggested that colonoscopy should be performed 12 months after surgery in case of a suspected surgical margin and that a further follow-up is no longer necessary if the margin is clear; meanwhile, transrectal EUS or MRI should be performed 12 months after resection if the status of lymph node metastases is unclear. In our study, 13 patients had a positive deep margin, among whom 3 underwent additional surgery, while the other 10 underwent a thorough follow-up. None were found to have tumor recurrence. We believe that all patients should be followed up after endoscopic resection for rectal NETs regardless of their tumor diameter. The follow-up approaches should involve endoscopy, ultrasound, blood biochemical examinations, and a chest and abdominal CT scan when necessary. The initial follow-up visit should be shortened for patients with tumors of positive margins, G2 grade, and larger than $10 \mathrm{~mm}$ in diameter. Recent studies have shown vascular invasion of small rectal NETs to be a risk factor for lymph node metastases. Despite the presence of vascular invasion, small rectal NETs treated by endoscopy have been found to have a good short-term outcome. However, it is not necessary to perform radical surgery immediately for small rectal NETs with vascular invasion, and a longterm follow-up is recommended (37). In short, the optimal strategy for the postoperative follow-up of rectal NETs warrants further exploration.

Our study also has certain limitations. First, this is a retrospective, single-center clinical study, and this might have led to potential selection bias in the comparison between modified EMR and ESD, which is inevitable in any retrospective study. Second, the sample size of our study was relatively small, and the conclusions of our study need to be validated by multicenter studies with a larger sample size. Finally, some patients only experienced a short followup time, and some did not complete a follow-up.

Since the methods for managing NETs remain controversial, especially for those $>10 \mathrm{~mm}$, the strength of our study is in the evaluation of the effect of modified EMR and ESD for NETs. We concluded that both modified EMR and ESD were safe and effective endoscopic approaches for the resection of rectal NETs $\leq 15 \mathrm{~mm}$ in diameter. The cost of ESD is higher than that of modified EMR and a much more complex procedure; our study provides further insight into how both patients and clinicians might decide between the different endoscopic approaches. Despite similar problems and shortcomings to previously published studies, including a limited sample size and retrospective design, our study has shown the novel impacts of modified EMR and ESD.

In conclusion, modified EMR and ESD are safe and effective for treating rectal NETs $\leq 15 \mathrm{~mm}$ in diameter. Endoscopic resection requires the comprehensive preoperative evaluation of certain risk factors, including the depth of tumor invasion and tumor diameter. The followup strategy for patients with rectal NETs after endoscopic resection needs to be further optimized.

\section{Acknowledgments}

We acknowledge the AME Editing Service for English language editing.

Funding: This work was supported by the National Natural Science Foundation of China (No. 82171698, 82170561, 81300279 and 81741067), the Scientific Research Project of Traditional Chinese Medicine Bureau of Guangdong Province (No. 20201010), the Natural Science Foundation for Distinguished Young Scholars of Guangdong Province (No. 2021B1515020003), and the Climbing Program of Introduced Talents and High-level Hospital Construction Project of Guangdong Provincial People's Hospital (No. DFJH201803, KJ012019099, KJ012021143, KY012021183).

\section{Footnote}

Reporting Checklist: The authors have completed the STROBE reporting checklist. Available at https://dx.doi. org/10.21037/jgo-21-391

Data Sharing Statement: Available at https://dx.doi. org/10.21037/jgo-21-391

Peer Review File: Available at https://dx.doi.org/10.21037/ jgo-21-391

Conflicts of Interest: All authors have completed the ICMJE uniform disclosure form (available at https://dx.doi. org/10.21037/jgo-21-391). The authors have no conflicts of interest to declare.

Ethical Statement: The authors are accountable for all aspects of the work in ensuring that questions related to the accuracy or integrity of any part of the work are appropriately investigated and resolved. The study was conducted in accordance with the Declaration of Helsinki (as 
revised in 2013). This study was approved by the Research Ethics Committee of Guangdong General Hospital (No. KY-Q-2021-184-01). Informed consent was waived because of the retrospective nature of the study, and anonymous clinical data was use.

Open Access Statement: This is an Open Access article distributed in accordance with the Creative Commons Attribution-NonCommercial-NoDerivs 4.0 International License (CC BY-NC-ND 4.0), which permits the noncommercial replication and distribution of the article with the strict proviso that no changes or edits are made and the original work is properly cited (including links to both the formal publication through the relevant DOI and the license). See: https://creativecommons.org/licenses/by-nc-nd/4.0/.

\section{References}

1. Eick J, Steinberg J, Schwertner C, et al. Rectal neuroendocrine tumors: endoscopic therapy. Chirurg 2016;87:288-91

2. Taghavi S, Jayarajan SN, Powers BD, et al. Examining rectal carcinoids in the era of screening colonoscopy: a surveillance, epidemiology, and end results analysis. Dis Colon Rectum 2013;56:952-9.

3. Gastrointestinal Pathology Study Group of Korean Society of Pathologists; Cho MY, Kim JM, et al. Current Trends of the Incidence and Pathological Diagnosis of Gastroenteropancreatic Neuroendocrine Tumors (GEPNETs) in Korea 2000-2009: Multicenter Study. Cancer Res Treat 2012;44:157-65.

4. Scherübl H. Rectal carcinoids are on the rise: early detection by screening endoscopy. Endoscopy 2009;41:162-5.

5. Soga J. Carcinoids of the rectum: an evaluation of 1271 reported cases. Surg Today 1997;27:112-9.

6. Kaminski M, Polkowski M, Regula J. Prevalence and endoscopic features of rectal neuroendocrine tumors (carcinoids) among 50148 participants of the Polish colorectal-cancer screening programme. Gut 2007;56:A310.

7. Zhou X, Xie H, Xie L, et al. Factors associated with lymph node metastasis in radically resected rectal carcinoids: a systematic review and meta-analysis. J Gastrointest Surg 2013;17:1689-97.

8. Yamagishi D, Matsubara N, Noda M, et al. Clinicopathological characteristics of rectal carcinoid patients undergoing surgical resection. Oncol Lett
2012;4:910-4.

9. Pape UF, Perren A, Niederle B, et al. ENETS Consensus Guidelines for the management of patients with neuroendocrine neoplasms from the jejuno-ileum and the appendix including goblet cell carcinomas. Neuroendocrinology 2012;95:135-56.

10. Jung ES, Kang YK, Cho MY, et al. Update on the proposal for creating a guideline for cancer registration of the gastrointestinal tumors (I-2). Korean J Pathol 2012;46:443-53.

11. Bertani E, Ravizza D, Milione M, et al. Neuroendocrine neoplasms of rectum: A management update. Cancer Treat Rev 2018;66:45-55.

12. Ahmed Y, Othman M. EMR/ESD: Techniques, Complications, and Evidence. Curr Gastroenterol Rep 2020;22:39.

13. Zheng JC, Zheng K, Zhao S, et al. Efficacy and safety of modified endoscopic mucosal resection for rectal neuroendocrine tumors: a meta-analysis. Z Gastroenterol 2020;58:137-45.

14. de Mestier L, Lepage C, Baudin E, et al. Digestive Neuroendocrine Neoplasms (NEN): French Intergroup clinical practice guidelines for diagnosis, treatment and follow-up (SNFGE, GTE, RENATEN, TENPATH, FFCD, GERCOR, UNICANCER, SFCD, SFED, SFRO, SFR). Dig Liver Dis 2020;52:473-92.

15. Basuroy R, Haji A, Ramage JK, et al. Review article: the investigation and management of rectal neuroendocrine tumours. Aliment Pharmacol Ther 2016;44:332-45.

16. Kim J, Kim JH, Lee JY, et al. Clinical outcomes of endoscopic mucosal resection for rectal neuroendocrine tumor. BMC Gastroenterol 2018;18:77.

17. Nagtegaal ID, Odze RD, Klimstra D, et al. The 2019 WHO classification of tumours of the digestive system. Histopathology 2020;76:182-8.

18. Tsai HJ, Wu CC, Tsai CR, et al. The epidemiology of neuroendocrine tumors in Taiwan: a nation-wide cancer registry-based study. PLoS One 2013;8:e62487.

19. Ko SH, Baeg MK, Ko SY, et al. Clinical characteristics, risk factors and outcomes of asymptomatic rectal neuroendocrine tumors. Surg Endosc 2017;31:3864-71.

20. Bang BW, Park JS, Kim HK, et al. Endoscopic Resection for Small Rectal Neuroendocrine Tumors: Comparison of Endoscopic Submucosal Resection with Band Ligation and Endoscopic Submucosal Dissection. Gastroenterol Res Pract 2016;2016:6198927.

21. Jeon JH, Cheung DY, Lee SJ, et al. Endoscopic resection yields reliable outcomes for small rectal neuroendocrine 
tumors. Dig Endosc 2014;26:556-63.

22. Duan X, Zhao M, Zhang S, et al. Effects of tumor distance from anal verge on survival outcomes for rectal NENs and lymphatic metastasis risk score for colorectal NENs. Int J Colorectal Dis 2020;35:1255-64.

23. Pavel M, Öberg K, Falconi M, et al. Gastroenteropancreatic neuroendocrine neoplasms: ESMO Clinical Practice Guidelines for diagnosis, treatment and follow-up. Ann Oncol 2020;31:844-60.

24. Caplin M, Sundin A, Nillson O, et al. ENETS Consensus Guidelines for the management of patients with digestive neuroendocrine neoplasms: colorectal neuroendocrine neoplasms. Neuroendocrinology 2012;95:88-97.

25. Nagata K, Tajiri K, Shimada S, et al. Rectal Neuroendocrine Tumor G1 with a Solitary Hepatic Metastatic Lesion. Intern Med 2017;56:289-93.

26. Naunheim KS, Zeitels J, Kaplan EL, et al. Rectal carcinoid tumors--treatment and prognosis. Surgery 1983;94:670-6.

27. Kunz PL, Reidy-Lagunes D, Anthony LB, et al. Consensus guidelines for the management and treatment of neuroendocrine tumors. Pancreas 2013;42:557-77.

28. Delle Fave G, O'Toole D, Sundin A, et al. ENETS Consensus Guidelines Update for Gastroduodenal Neuroendocrine Neoplasms. Neuroendocrinology 2016;103:119-24.

29. Perren A, Couvelard A, Scoazec JY, et al. ENETS Consensus Guidelines for the Standards of Care in Neuroendocrine Tumors: Pathology: Diagnosis and Prognostic Stratification. Neuroendocrinology 2017;105:196-200.

30. Shah MH, Goldner WS, Halfdanarson TR, et al. NCCN Guidelines Insights: Neuroendocrine and Adrenal Tumors, Version 2.2018. J Natl Compr Canc Netw

Cite this article as: Zheng Y, Guo K, Zeng R, Chen Z, Liu W, Zhang X, Liang W, Liu J, Chen H, Sha W. Prognosis of rectal neuroendocrine tumors after endoscopic resection: a singlecenter retrospective study. J Gastrointest Oncol 2021;12(6):27632774. doi: 10.21037/jgo-21-391
2018;16:693-702.

31. Park SB, Kim DJ, Kim HW, et al. Is endoscopic ultrasonography essential for endoscopic resection of small rectal neuroendocrine tumors? World J Gastroenterol 2017;23:2037-43.

32. Yang DH, Park Y, Park SH, et al. Cap-assisted EMR for rectal neuroendocrine tumors: comparisons with conventional EMR and endoscopic submucosal dissection (with videos). Gastrointest Endosc 2016;83:1015-22; quiz 1023-.e6.

33. Wang XY, Chai NL, Linghu EQ, et al. The outcomes of modified endoscopic mucosal resection and endoscopic submucosal dissection for the treatment of rectal neuroendocrine tumors and the value of endoscopic morphology classification in endoscopic resection. BMC Gastroenterol 2020;20:200.

34. Sung HY, Kim SW, Kang WK, et al. Long-term prognosis of an endoscopically treated rectal neuroendocrine tumor: 10-year experience in a single institution. Eur J Gastroenterol Hepatol 2012;24:978-83.

35. Chung HG, Goh MJ, Kim ER, et al. Recurrence pattern and surveillance strategy for rectal neuroendocrine tumors after endoscopic resection. J Gastroenterol Hepatol 2021;36:968-73.

36. Singh S, Moody L, Chan DL, et al. Followup Recommendations for Completely Resected Gastroenteropancreatic Neuroendocrine Tumors. JAMA Oncol 2018;4:1597-604.

37. Kang HS, Kwon MJ, Kim TH, et al. Lymphovascular invasion as a prognostic value in small rectal neuroendocrine tumor treated by local excision: A systematic review and meta-analysis. Pathol Res Pract 2019;215:152642. 